\title{
Intracavity Modulation of THz p-Ge Laser Gain by Interband Optical Excitation
}

\author{
C.J. Fredricksen 1 , A.V. Muravjov², and R.E. Peale 2 \\ 1Zaubertek, Inc., 12565 Research Pkwy. Suite 300, Orlando, FL 32826 \\ 2Department of Physics, University of Central Florida, Orlando, FL 32816
}

\begin{abstract}
Optical quenching of the THz inter-sub-band p-Ge laser (tunable in the wavelength range 70-200 $\mu \mathrm{m}$ with $\sim 1 \mathrm{~W}$ output power) by Nd:YAG laser radiation has been investigated. YAG laser pulses were coupled into a p-Ge laser cavity through a $\mathrm{SrTiO}_{3}$ laser mirror, which is highly reflecting at cryogenic temperatures for $\mathrm{THz}$ frequencies and transparent for visible and near-IR light. Fast quenching of the p-Ge laser emission intensity was observed and attributed to free carrier absorption by optically generated electron-hole pairs in a thin layer of the active p-Ge crystal end surface. The effect also occurs when the interband absorption is confined to optically stimulated intracavity $\mathrm{Si}$ or GaAs spacers, which are transparent in the far-IR, placed between the $\mathrm{SrTiO}_{3}$ laser mirror and the active crystal end face. Such fast quenching of the p-Ge laser might be used to sharpen the trailing edge of the far-IR emission pulse for time-resolved or cavity-ring-down spectroscopic applications. Direct-gap semiconductor spacers might be used as fast, optically controlled intracavity modulators for active mode-locking.
\end{abstract}

\section{INTRODUCTION}

The p-Ge laser is tunable throughout the 1.5 to $4.2 \mathrm{THz}$ region (Fig. 1a) with watt-level output power [1]. The laser mechanism is based on population inversion between light- and heavy-hole valence sub-bands in crossed electric and magnetic fields. The broad gain bandwidth of the p-Ge laser medium can support generation of pulses shorter than $10 \mathrm{ps}[2]$. Sub-ns pulses of far-IR radiation have been generated by passive[3] or active [4] mode locking, where the gain is modulated by an RF electric field at a frequency equal to the laser cavity roundtrip frequency applied to small ohmic contacts. The shortest pulses we have observed were $\sim 50$ ps duration [3], limited by our transient digitizer. The pulse train of a harmonically mode-locked p-Ge laser can be electrically modulated (Fig. 1b) [5]. However, active mode-locking of $p$-Ge lasers has required large rf power because of the low impedance of the active crystal. The associated power electronics are incompatible with practical applications. One aspect of this work is to explore the possibility that fast optical perturbation of the p-Ge laser gain might be a more convenient method of active mode-locking.

The primary focus of this work is to test the degree to which optical perturbation can sharpen the trailing edge of the $\mathrm{p}$-Ge laser emission pulse. The time scale of this trailing edge is usually on the order of $100 \mathrm{~ns}$ to $1 \mu \mathrm{s}$ and is determined either by the speed of the excitation electronics or by the over-heating of the Ge crystal during electrical excitation. The interest in a fast turn off is the possibility of developing a far-IR Cavity Ring Down Spectrometer (CRDS). This technique detects trace concentrations of gases by the effect their absorption has on increasing the decay time of electromagnetic energy stored in a passive optical cavity. Due to the unavailability of far-IR mirrors with reflectivity better than $\sim 99 \%$, typical passive cavity lifetimes are less than $1 \mu \mathrm{s}$. Hence, a laser source that pumps such a cavity in a CRDS system must be able to be shut off in much less than $1 \mu \mathrm{s}$. 

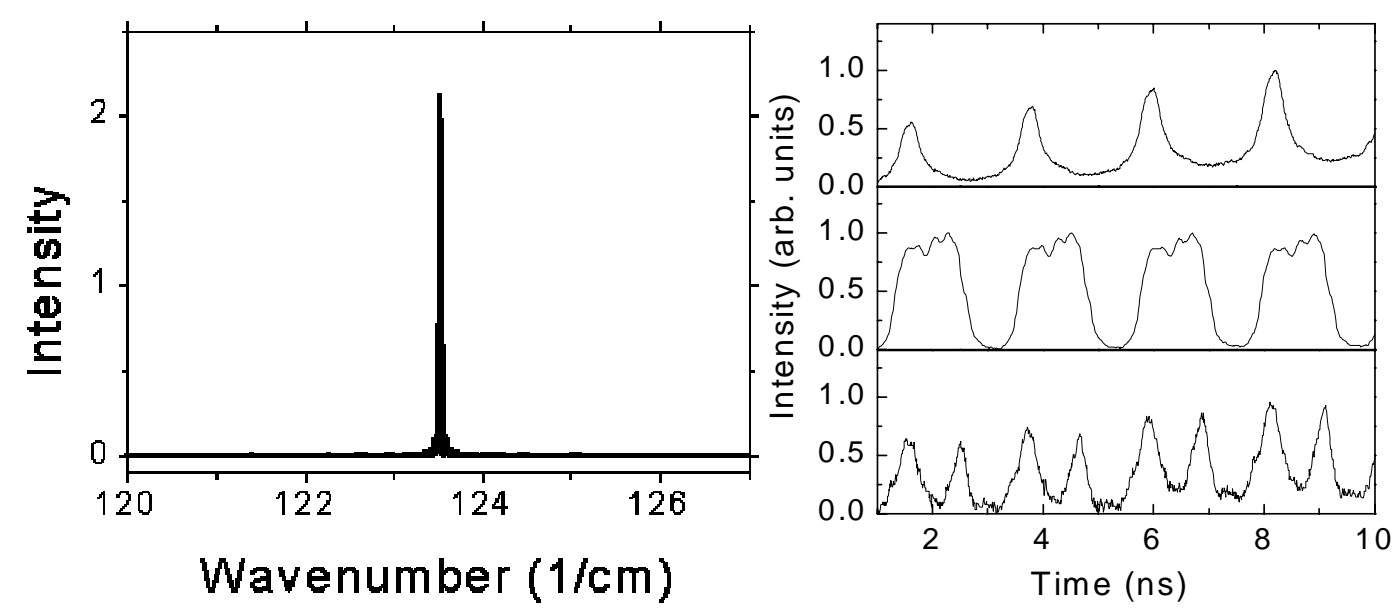

Figure 1 - p-Ge laser functionality. a) The p-Ge laser is tunable throughout the region $50-140 \mathrm{~cm}^{-1}(70-200 \mu \mathrm{m}, 1.5-4.2$ THz). b) A harmonically mode-locked p-Ge laser has been electronically mode-locked to yield sub-ns pulses of far-IR radiation with variable pulse position.

\section{EXPERIMENTAL}

Figure 2 presents a schematic of the experimental setup. Germanium laser rods were cut from gallium-doped single crystals to the shape of rectangular parallelepipeds with approximate dimensions $50 \mathrm{~mm} \times 7 \mathrm{~mm} \times 5 \mathrm{~mm}$. Small end faces were polished flat and parallel to within 30 arcsec. $\mathrm{SrTiO}_{3}$ flats were attached to the end faces as laser mirrors. The crystal was placed at the center of a superconducting solenoid and inserted into a liquid helium storage dewar. Electric field pulses were applied to ohmic contacts on the narrow lateral faces of the active crystal using a thyratron. Far-IR laser radiation was detected using Ge:Ga photodetectors $\left(\mathrm{N}_{\mathrm{Ga}}=10^{14} \mathrm{~cm}^{-3}\right.$, temporal resolution of $50-100 \mathrm{~ns} ; \mathrm{N}_{\mathrm{Ga}}=10^{15} \mathrm{~cm}^{-3},<5 \mathrm{~ns}$ temporal resolution) in the same dewar. Data was recorded using a $200 \mathrm{MHz}$ storage oscilloscope or a Tektronix SCD5000 transient digitizer with $5 \mathrm{GHz}$ bandwidth.

Radiation from a Q-switched Nd:YAG laser $(532 \mathrm{~nm}$ x $0.5 \mathrm{~mJ}$ x $8 \mathrm{~ns}$ and $1064 \mathrm{~nm}$ x $1.5 \mathrm{~mJ}$ x $10 \mathrm{~ns}$ ) was directed onto the end face of the laser crystal through an optical fiber bundle coupled to a quartz rod, whose end was positioned just above the $\mathrm{SrTiO}_{3}$ back mirror of the laser cavity. A GaAs detector monitored the timing of the YAG pulse.

Passive GaAs or Si plates were placed between the $\mathrm{SrTiO}_{3}$ back mirror and the end face of the active p-Ge crystal. Both materials have been tested in the p-Ge laser cavity and have been shown to have a high transparency [6]. 


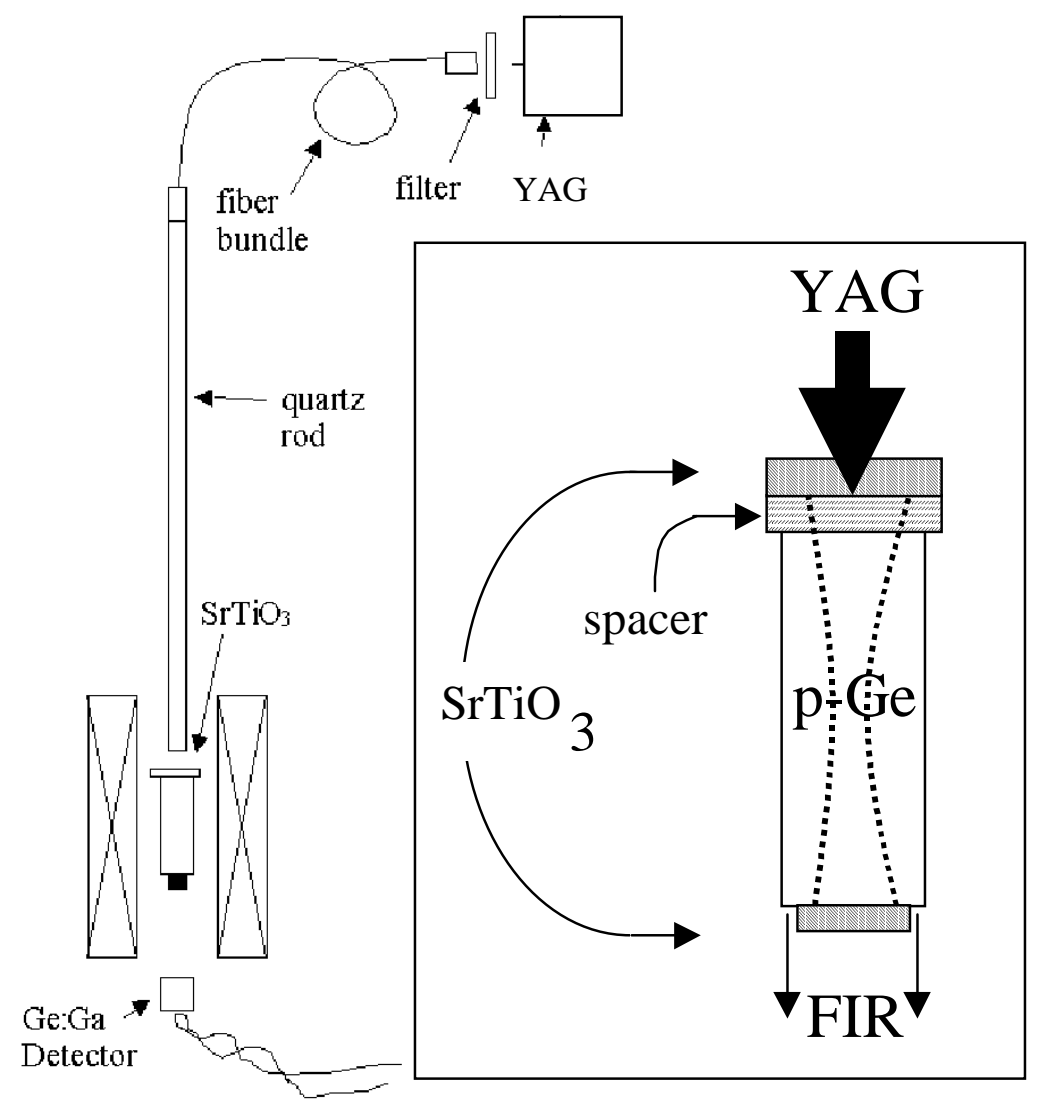

Figure 2 - Experimental setup for optical modulation of p-Ge laser. The p-Ge active crystal is positioned within a superconducting solenoid. The quartz rod is held just above the back mirror of the laser cavity. The Ge:Ga detector is positioned at the bottom of the dewar. Details of the p-Ge laser cavity construction are shown in the box. YAG laser radiation is absorbed in a thin layer at the end of the p-Ge active crystal or by a Si or GaAs spacer (when a spacer was used).

\section{RESULTS}

Fig. 3 shows the timing characteristics and typical results for a YAG pulse incident directly on the end face of the active crystal, as shown in the box in Figure 2 (minus the intracavity spacer). The bottom plot shows a $1.6 \mu \mathrm{s}$ current pulse applied to the laser rod. A few percent increase in current through the active crystal occurs when laser radiation is present due to fundamental absorption. The middle plot presents the p-Ge laser emission pulse, which begins rising about $600 \mathrm{~ns}$ after the beginning of the current pulse. The rapid decrease and subsequent recovery of the p-Ge emission pulse coincident with the YAG laser perturbation (upper plot) is evident. The effect of the YAG pulse is to temporarily quench the p-Ge laser emission. The observed temporal profile is detector limited. The observed quenching effect was very similar whether $532 \mathrm{~nm}$ or $1064 \mathrm{~nm}$ YAG laser radiation was used though the $1064 \mathrm{~nm}$ fundamental was more effective, presumably because of higher available power. 


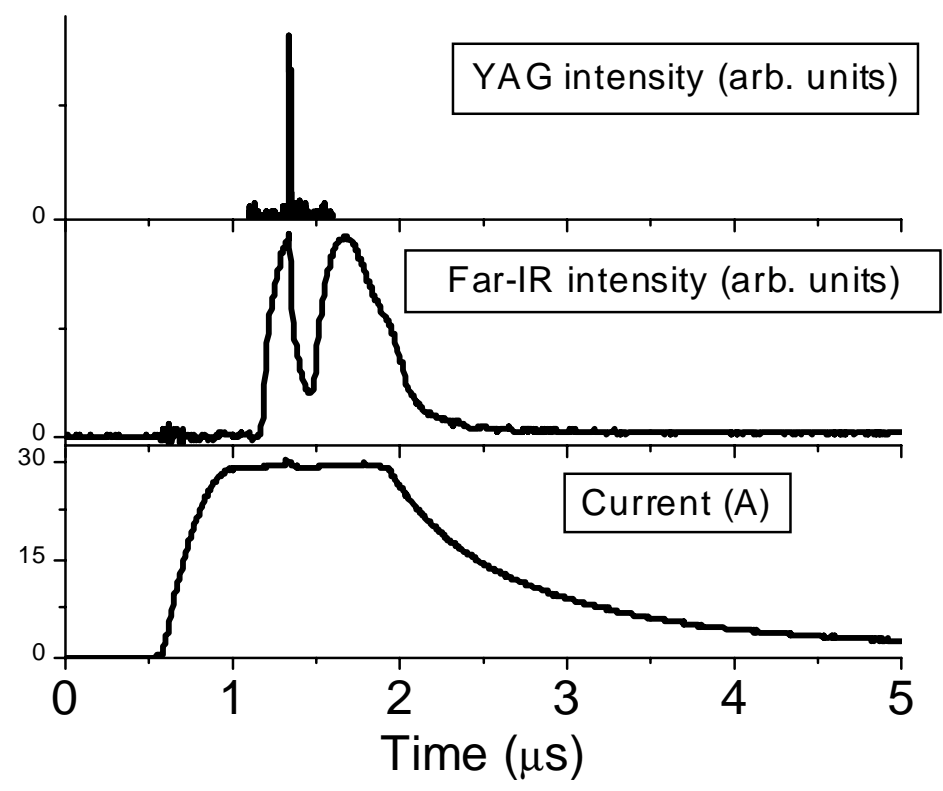

Figure 3 - Timing characteristics of the optical modulation experiments (slow far-IR detector). The YAG pulse energy (incident directly on the end face of the crystal without an intracavity spacer in place) was moderate, to allow recovery of the p-Ge laser emission.

Figure 4 shows the p-Ge laser emission pulse with increasing delay of the $532 \mathrm{~nm}$ YAG pulse. Quenching was not observed when the YAG pulse came more than 100 ns before the current pulse.

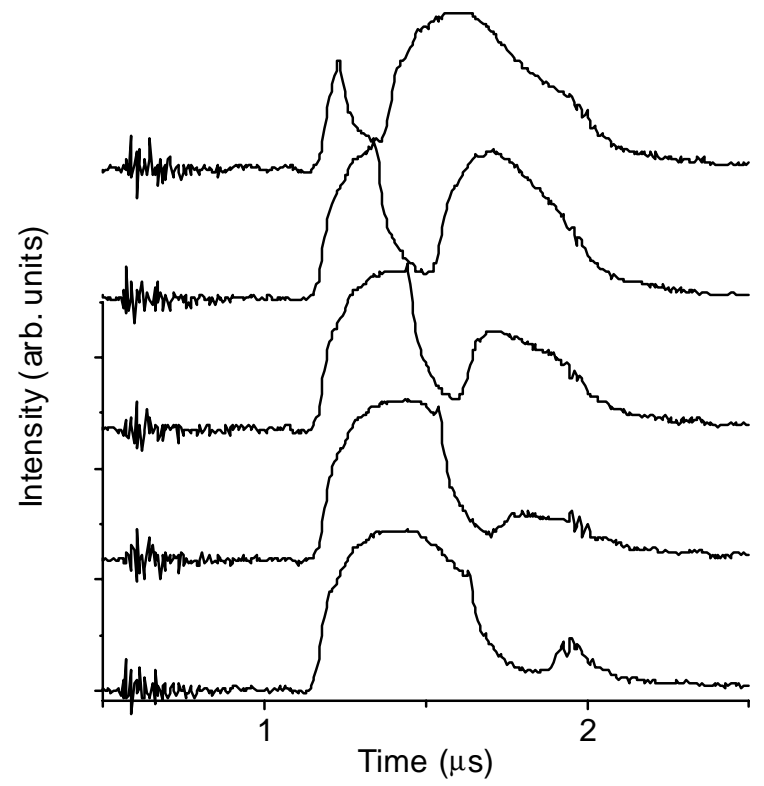

Figure 4 - p-Ge laser emission with increasing (top to bottom) delay of the YAG laser excitation (slow detector). The ringing in the $\mathrm{p}-\mathrm{Ge}$ laser emission trace is electromagnetic interference from the fast current perturbation.

Figure 5 shows the quenching with moderate YAG pulse energy at higher temporal resolution, using the fast detector. Increasing the YAG pulse energy can lead to quenching of the far-IR emission for the remainder of the excitation current pulse, as seen in Figure 6. Using the fast detector and $5 \mathrm{GHz}$ transient digitizer, quenching is 
observed to occur over time scales on the order of a few nanoseconds, as seen in figure 7 . This value is still detector limited. The recovery of far-IR emission, on the other hand, takes $\sim 100 \mathrm{~ns}$.

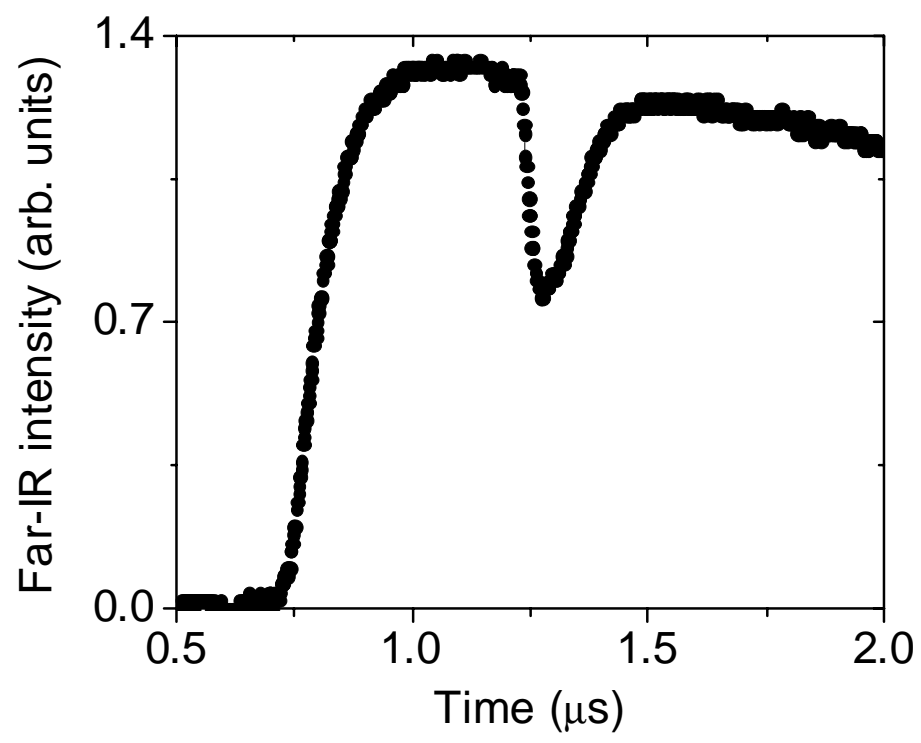

Figure 5 - Fast quench and slow recovery of far-IR p-Ge laser emission as a result of YAG laser irradiance of Ge end face (fast detector, $200 \mathrm{MHz}$ scope). After quenching, the rate of signal recovery is comparable to the initial rise in emission.

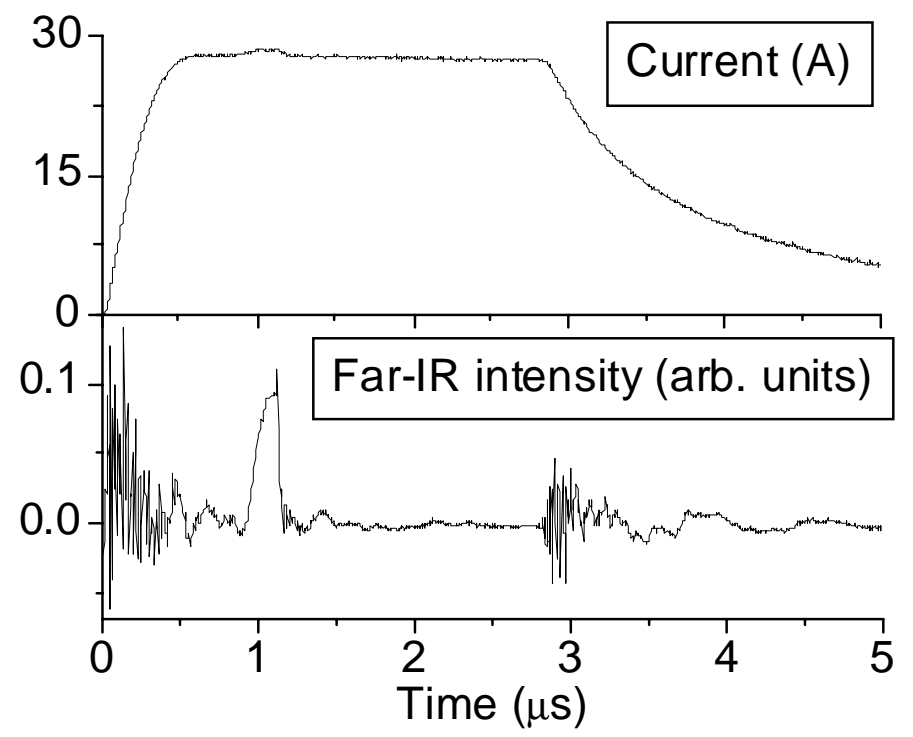

Figure 6 - Far-IR emission quenched for the remainder of the electric field pulse due to higher YAG pulse energy (fast detector, $200 \mathrm{MHz}$ oscilloscope). 


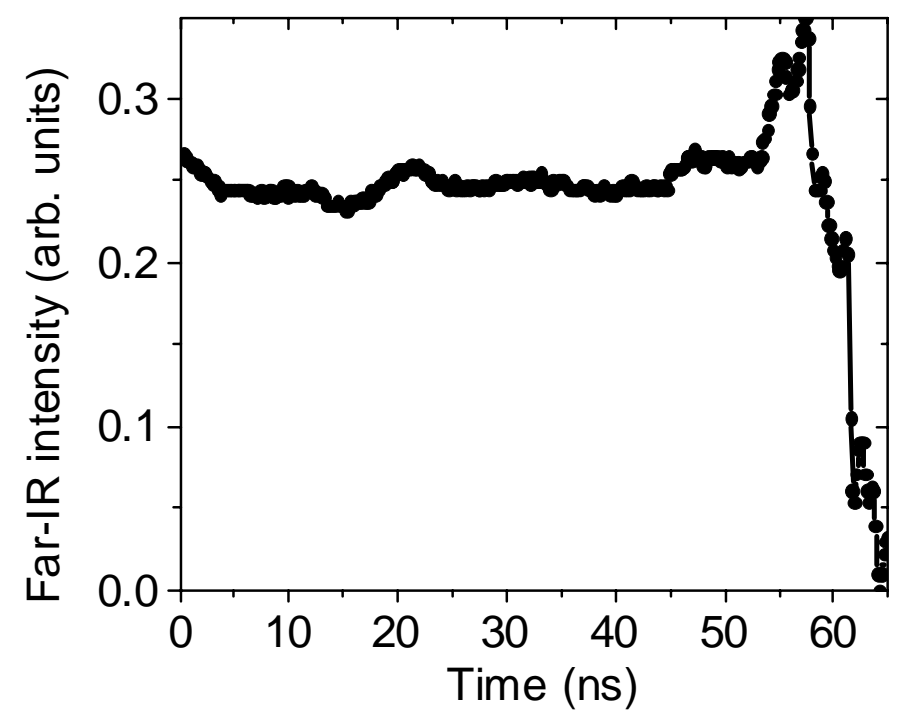

Figure 7 - Quenching of far-IR emission measured with fast transient digitizer and detector.

In an attempt to speed up the quenching and/or recovery, a passive $\mathrm{GaAs}$ or Si plate was placed between the $\mathrm{SrTiO}_{3}$ back mirror and the end face of the active p-Ge crystal. The excitation wavelength for a GaAs modulator needs to be shorter than $815 \mathrm{~nm}$, so doubled YAG at $532 \mathrm{~nm}$ was used. YAG excitation at $1064 \mathrm{~nm}$ had essentially the same effect as seen without the spacer, as GaAs is transparent to this radiation.

Figure 8 shows a p-Ge laser emission curve with a GaAs spacer present and moderate YAG pulse energy applied. A fast quenching time on the order of the duration of the incident YAG pulse (several ns) can be seen. This data was taken using the fast Ge:Ga detector. The recovery is still slow.

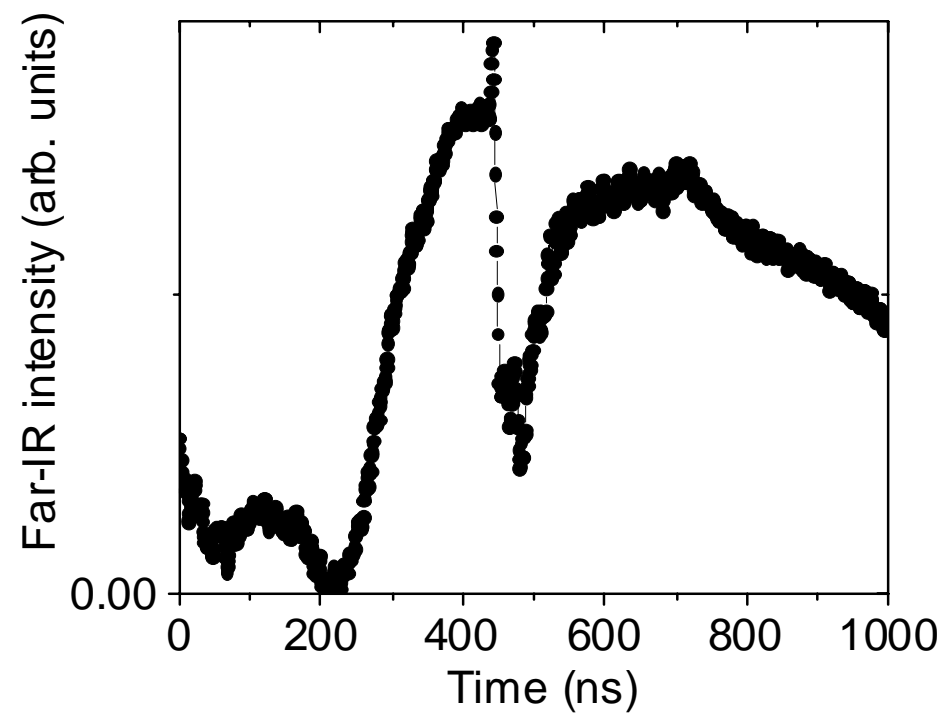

Figure 8 - Fast quench of p-Ge laser with a Ga As spacer excited by $532 \mathrm{~nm}$ laser pulse (fast detector).

A weak thermal effect is observed only when an intracavity spacer is irradiated. Figure 9 shows the thermal effect with a thin $(<1 \mathrm{~mm}) \mathrm{Si}$ spacer present in the cavity. The graph shows the resulting delay of the leading edge of the pGe laser emission vs. the delay between the modulating pulse and the current pulse. This effect was not observed without an intracavity spacer present, presumably because of fast diffusion of the heat generated in the upper $\sim \mu \mathrm{m}$ layer into the large bulk active crystal at $4 \mathrm{~K}$. 


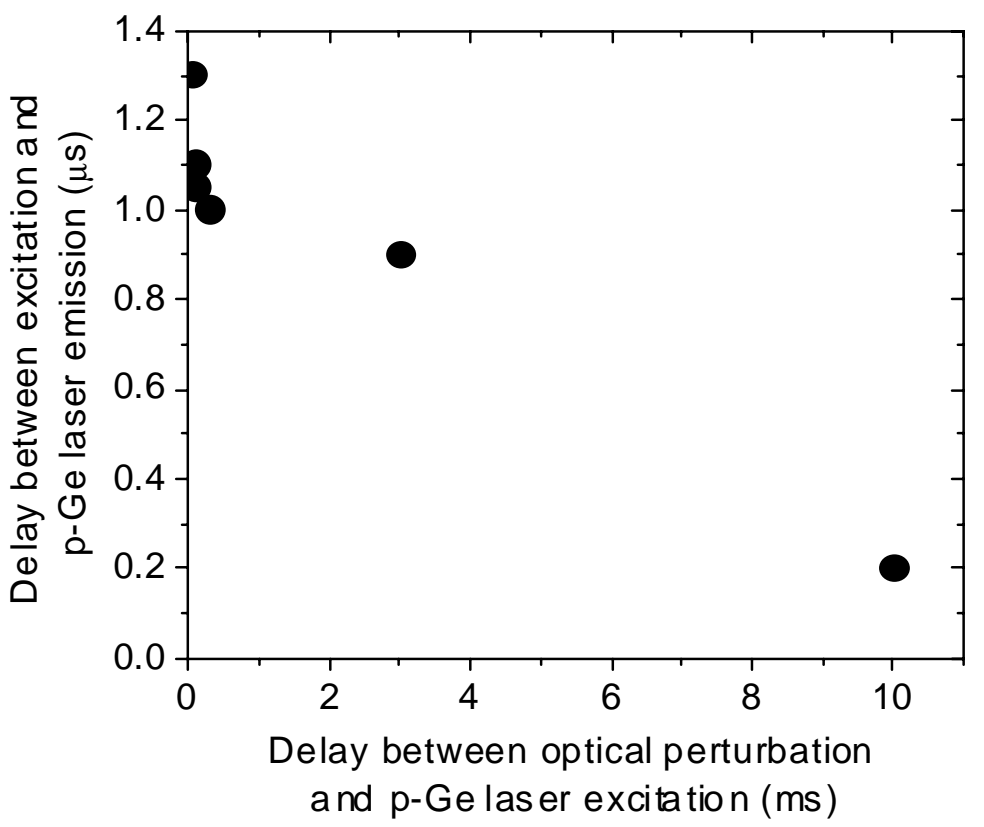

Figure 9 - Thermally induced delay in appearance of p-Ge emission when Si intracavity spacer is perturbed by YAG laser pulse.

\section{DISCUSSION}

Based on our observations of quenching using the fast Ge detector and $5 \mathrm{GHz}$ transient digitizer, we believe that quenching can occur within the typical p-Ge laser cavity round trip time (1 ns) for sufficiently strong YAG pulse energy. Furthermore, the end of the far-IR laser emission pulse can be completely extinguished with appropriate YAG pulse energy for cavity ringdown spectroscopic applications. The recovery of far-IR emission may also be prevented by switching off the $\mathrm{p}$-Ge laser excitation current. For lower YAG pulse energies, the recovery times observed without a spacer are too slow for direct optical modulation of the active crystal to be useful for active mode-locking by gain modulation. Recovery is limited by a combination of the lifetime of photogenerated e-h pairs and the population inversion buildup dynamics. While the population inversion buildup time is typically $\sim 100 \mathrm{ps,}$ the lifetime of photogenerated e-h pairs in the indirect gap semiconductor germanium at $4 \mathrm{~K}$ is long.

When the near-IR/visible YAG radiation is incident on the end face of the active p-Ge crystal or on passive spacers, e-h pairs are created in a thin layer just beneath the $\mathrm{SrTiO}_{3}$ mirror. One effect that contributes to the quenching is that the e-h pairs introduce free carrier absorption, obscuring the mirror and decreasing the cavity Q. Also of major impact is the disruption of the uniformity of the electric field at the end of the crystal caused by the generation of e-h pairs. The spacer experiments were useful in helping to determine the impact of electric field non-uniformity. The fact that quenching was still observed with passive spacers indicates that free carrier absorption is sufficient to quench the lasing and that the disruption of the electric field uniformity at the end face of the crystal is not the main contributing factor.

It is unlikely that the observed fast quenching is a thermal effect because the observed time scales are much faster than thermal time constants for the large active crystal. The fact that quenching was not observed when the YAG pulse came more than $100 \mathrm{~ns}$ before the current pulse (without a spacer) further indicates that the observed quenching is not a thermal effect. Weak additional thermal effects were observed, however, when an intracavity element was present. When the YAG laser emission was absorbed by the thin spacer, there was no large bulk for the generated heat to diffuse into. The small temperature rise created in the spacer induces loss from phonon absorption for the circulating far-IR radiation. 
The e-h pair lifetime is shorter in the direct-gap GaAs than in the indirect Ge. Hence, it was hypothesized that the use of a GaAs spacer might increase the speed of recovery if pair lifetime is the important factor. In GaAs, the electron-hole recombination time is short and the p-Ge laser emission recovery time could be on the order of the gain recovery time (1 ns). The recovery observed with a GaAs spacer present was still slow. Evidently, when the modulating pulse is of sufficient strength and duration, the p-Ge laser stimulated emission is reduced to the level of spontaneous emission. The far-IR emission recovery, therefore, is dependent upon the re-emergence of stimulated emission; i.e. it is dependent upon the gain. In this case, the recovery of far-IR emission should take approximately $100 \mathrm{~ns}$, as seen at the beginning of the far-IR pulse (after the initiation of the excitation current pulse).

\section{CONCLUSIONS}

Near-IR/visible radiation, incident on one end face of the active p-Ge laser crystal or on a passive GaAs or Si spacer, can quench the laser emission in just a few ns or less. This feature may have applications in far-infrared timeresolved or cavity ring-down spectroscopies. It is particularly attractive for CRDS, as the advent of mirrors with high reflectivity in the far-IR may allow ultra-trace gas detection at wavelengths longer than $10 \mu \mathrm{m}$ for the first time. This underutilized region of the electromagnetic spectrum is rich in characteristic absorption lines based on rotor and vibrational transitions. Fast optical intraband excitation of a direct-gap spacer such as GaAs in the p-Ge cavity, with typical round trip times on the order of $1 \mathrm{~ns}$, may also be a means of active mode-locking (with a cavity of sufficient length) to produce ps pulses of far-infrared radiation. Using fast-modulated near-IR laser radiation, one might realize a harmonically mode-locked $\mathrm{THz}$ laser with pulse-separation modulation as a novel means of freespace telemetry on a $\mathrm{THz}$ carrier.

\section{REFERENCES}

1. "Special Issue on Far-infrared Semiconductor Lasers," edited by E. Gornik and A.A.Andronov, Opt. Quantum Electron. 23 (1991).

2. R.C.Strijbos, J.G.S.Lok, and W.Th.Wenckebach, "A Monte Carlo simulation of mode-locked hot-hole laser operation," J.Phys.Condens.Matter, 6, 7461-7468 (1994).

3. A. V. Muravjov, R. C. Strijbos, C. J. Fredricksen, H. Weidner, W. Trimble, S. H. Withers, S. G. Pavlov, V. N. Shastin, and R. E. Peale, "Evidence for self-mode-locking in p-Ge laser emission," Appl. Phys. Lett. 73,3037 3039 (1998).

4. A. V. Muravjov, S. H. Withers, R. C. Strijbos, S. G. Pavlov, V. N. Shastin, and R. E. Peale, "Actively modelocked p-Ge laser in Faraday configuration," Appl. Phys. Lett. 75, 2882 - 2884 (1999).

5. A. V. Muravjov, R. C. Strijbos, C. J. Fredricksen, S. H. Withers, W. Trimble, S. G. Pavlov, V. N. Shastin, and R. E. Peale, "Pulse separation control for mode-locked far-infrared p-Ge lasers," Appl. Phys. Lett. 74, 167 - 169 (1999).

6. E. W. Nelson, S. H. Withers, A. V. Muravjov, R. C. Strijbos, R. E. Peale, S. G. Pavlov, V. N. Shastin, and C. J. Fredricksen, "High resolution study of composite cavity effects for p-Ge lasers," J. Quantum Electronics 37, 1525 - 1530 (2001). 